\title{
Recruited peripheral blood monocytes participate in the liver extramedullary hematopoietic milieu*
}

\author{
Ayako Nakamura-Ishizu, Shunichi Morikawa, Kazuhiko Shimizu, and Taichi Ezaki \\ Department of Anatomy and Developmental Biology, Tokyo Women's Medical University, Tokyo, Japan
}

\begin{abstract}
Summary. The hematopoietic microenvironment has been investigated and well defined in the bone marrow. However, there is a lack of studies on the extramedullary hematopoietic milieu such as in the liver, to which hematopoietic stem cells migrate and there commence hematopoiesis under pathological conditions such as bone marrow failure. We induced extramedullary hematopoiesis by phenylhydrazine in the adult mouse liver and investigated the immunohistochemical, ultrastructural, and molecular changes within this organ. Using an intravital lectin injection technique, we found numerous monocytes attached to the central vein prior to hematopoietic foci formation. These cells were later incorporated into the hematopoietic foci. An increase in the mRNA expressions of the monocyte attracting chemokine CCL-2 (MCP1) was noted in the central vein region as well as in cells within the hematopoietic foci. Together with local liver components, we regard these monocytes as components of the extramedullary hematopoietic milieu. We conclude that the recruitment of extra-hepatic monocytes is an important event during extramedullary hematopoiesis in the liver and that these monocytes participate in the liver hematopoietic microenvironment.
\end{abstract}

Received December 22, 2010

* This work has been supported by the Itoe Okamoto Scientific Award from Shiseikai.

Address for correspondence: Prof. Taichi Ezaki, MD, PhD, Department of Anatomy and Developmental Biology, Tokyo Women's Medical University, 8-1 Kawada-cho, Shinjuku-ku, Tokyo, 162-8666, Japan

Tel \& Fax: +81-3-5269-7407

Email: ezakit@research.twmu.ac.jp

\section{Introduction}

The bone marrow is the major hematopoietic organ maintaining homeostasis of mature hematopoietic cells during adulthood. A specialized microenviroment supports the development and maturation of hematopoietic cells (Schofield, 1978). In the bone marrow, osteoblasts (Taichman, 2005), macrophages (Hanspal and Hanspal, 1994), sinusoidal endothelial cells (Kopp et al., 2005), reticular cells (Penn et al., 1993), fibroblasts (Zuckerman et al., 1985) and adipocytes (Yin and $\mathrm{Li}, 2006$ ) comprise as the hematopoietic milieu. Organs other than the bone marrow are known to participate in hematopoiesis. Hematopoietic stem cells (HSCs) enter the circulation from the bone marrow at certain rates homeostatically (Wright et al., 2001) and can lodge in the embryonic hematopoietic tissues such as the liver and spleen for hematopoiesis during bone marrow failure (O'Keane et al., 1989), severe anemia (Taher et al., 2006), or myeloproliferative diseases (Ahmed and Chang, 2006). However, the hematopoietic microenvironment in these extramedullary sites remains poorly defined.

Macrophages are known as a crucial component of the hematopoietic microenvironment (Weiss, 1976). A central macrophage surrounded by erythroid cells composes erythroblastic islands as a functional unit for erythroid hematopoiesis (Chasis, 2006). The phenotype and functions of the central macrophage in the erythroblastic islands have been well investigated in the bone marrow (Lee et al., 1988). However, the origin and phenotype of the central macrophages during liver extramedullary hematopoiesis is unclear. Some reports have speculated that Kupffer cells in the liver undergo phenotypic changes to nurse hematopoietic cells during extramedullary hematopoiesis (de Jong et al., 1994; Klein et al., 2007). Identification of the origin and signals that recruit and activate these macrophages is necessary to further 
understand hematopoietic stem cell biology.

We here show that, during extramedullary hematopoiesis in the adult mouse liver, massive numbers of peripheral monocytes infiltrate into the liver. Our evidence clearly indicates that the origin of some macrophages in the erythroblastic islands formed in the adult liver is extra-hepatic. These recruited monocytes then participate in the extramedullary hematopoietic milieu in the liver.

\section{Materials and Methods}

\section{Animal}

Male BALB/c mice, $8-10$ weeks of age, were purchased from Japan SLC Inc. (Shizuoka). They were maintained in air filtered clean rooms and fed with sterilized standard laboratory chow and water at the Institute of Laboratory Animals, Tokyo Women's Medical University. The following experiments were conducted in accordance with the regulations of the Institute of Laboratory Animals for Animal Experimentation at Tokyo Women's Medical University. On use, all mice were anesthetized by an intramuscular injection of a mixture of ketamine $(87 \mathrm{mg}$ / $\mathrm{kg}$ body weight) and xylazine (13 $\mathrm{mg} / \mathrm{kg}$ body weight).

\section{Induction of extramedullary hematopoiesis in the liver by phenylhydrazine $(\mathrm{PHZ})$ in splenectomized mice}

Extramedullary hematopoiesis in the liver was induced with phenylhydrazine hydrochloride (PHZ: SigmaAldrich, St. Louis, MO, USA) according to the method proposed by Adler S et al. (Adler and Trobaugh, 1978) with some modifications (Fig.1). Briefly, mice were splenectomized 7-10 days before PHZ injection. Subcutaneous injection of $1.2 \mathrm{mg}$ PHZ in $0.1 \mathrm{ml}$ phosphate buffered saline solution (PBS: Fuso Chemicals, Osaka) was performed for three consecutive days. Control animals were splenectomized animals injected with $0.1 \mathrm{~m} l$ of buffered saline subcutaneously. The first injection day was denoted as day 0 . Animals were dissected on days 2 , $4,7,14$ and 21 .

\section{Intravital lectin staining and perfusion fixation}

In order to delineate blood vessel endothelial cells and monocytes, an intravital lectin injection was performed prior to perfusion fixation of the animals. FITCconjugated Ricinus communis agglutinin-I (RCA-I) (200 $\mu l$; Vector Laboratories Inc., Burlingam, USA), diluted in PBS at a concentration of $0.5 \mathrm{mg} / \mathrm{ml}$, was injected individually into the tail vein of the mice. Ten minutes after injection, the animals were fixed by perfusion of $4 \%$ paraformaldehyde (PFA) in PBS through the left ventricle for 5-10 min at pressures ranging from 120 to $80 \mathrm{mmHg}$. Their livers were removed and postfixed in 4\% PFA in PBS at room temperature (RT) for 1 h. After washing with PBS and immersion in $30 \%$ sucrose in PBS, the organs were embedded in Tissue-Tek OCT compound (Sakura Finetek, Torrance, CA, USA) and snap frozen in liquid nitrogen for cryosection.

\section{Immunohistochemistry}

Cryosections (16 $\mu \mathrm{m}$ thick) of the organs were immersed in a $1 \%$ Block-ace blocking solution (Dainippon Seiyaku Ltd., Tokyo) at RT for $15 \mathrm{~min}$. The sections were then treated with primary antibodies to Type IV collagen (LSL CO., Tokyo), LYVE-1 (AngioBio, CA, USA) or CD45 (PharMingen, NJ, USA) and corresponding fluorescent conjugated secondary antibodies (Jackson ImmunoResearch, PA, USA). Thereafter, the sections were fixed with $4 \%$ PFA for $10 \mathrm{~min}$ and mounted in Vectashield (Vector Laboratories Inc.). The fluorescent images were observed under a Leica TCS-SL confocal laser scanning microscope (Leica, Wetzlar, Germany). Concomitantly, hematoxy-eosin stained samples were made and observed under an Olympus BX-51 light microscope (Olympus, Tokyo).

For bright field immunohistochemistry, sections were reacted with either F4/80 (Serotec, Kidlington, UK) or desmin (Abcam, Cambridge, UK) antibodies, followed by their corresponding peroxidase conjugated secondary antibodies. A peroxidase reaction was developed at $\mathrm{RT}$ for $10 \mathrm{~min}$ in a reaction mixture of $10 \mathrm{mg}$ of 3'-diaminobenzidine hydrochloride (DAB: Dojin Chemicals, Kumamoto) and $10 \mu l$ of $30 \% \mathrm{H}_{2} \mathrm{O}_{2}$ in $50 \mathrm{~m} l$ of PBS. The sections were counterstained with hematoxylin.

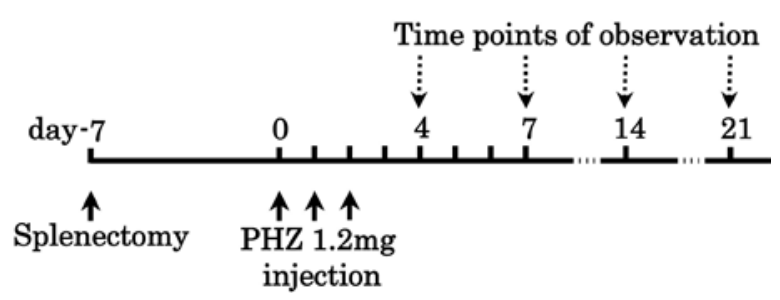

Fig. 1. Protocol of extramedullary hematopoieis. 


\section{PKH26 labeling and transplantation of peripheral blood monocytes}

In order to determine the origin of the recruited cells that appeared during extramedullary hematopoiesis, we designed a cell transfer experiment with various combinations of donor cells and host mice (Fig. 2). We first labeled phagocytic cells in the peripheral blood from either control (splenectomized mice without PHZ treatment) or day 4 mice and transplanted them individually into recipient mice either under or not under the same hemolytic stress. Donor mice were anesthetized and opened through the abdomen. Whole

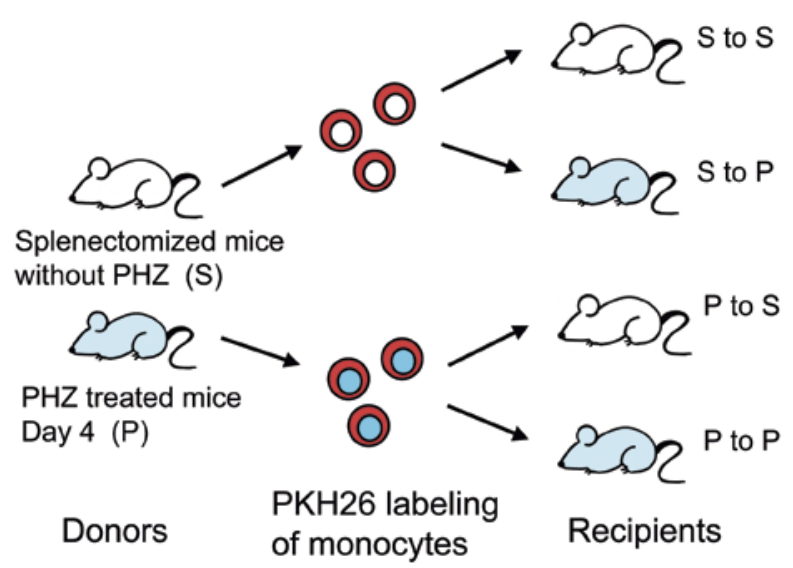

Fig. 2. Transplantation protocol of PKH26 labeled monocytes. blood samples were obtained from the inferior vena cava using a $23 \mathrm{G}$ needle. A PKH26 red fluorescent cell linker kit (Sigma-Aldrich Co.) was used for monocyte labeling according to the manufacturer's manual. Labeled cells were resuspended in calcium and magnesium-free PBS at a concentration of $4 \times 10^{8}$ cells $/ \mathrm{m} l$ and $100 \mu l$ of this solution was transfused into the tail vein of the recipient mice. Livers of the perfusion fixed mice were obtained $4 \mathrm{~h}$ and 3 days (equivalent to day 7 of extramedullary hematopoiesis protocol) after the transplantation. For 4h samples, the number of PKH26 positive cells per field at a magnification of 200 times was counted. For 3-day samples, the ratios of hematopoietic foci that contained at least one PKH26 positive cell to the total focus numbers were calculated for 100 foci per sample.

\section{Semi-quantitative reverse transcriptase-polymerase chain reaction (RT-PCR) for chemokines CCL-2 and CCL-5}

Splenectomized mice treated with PHZ were sacrificed by cervical dislocation on days 2, 4 and 7. Total RNA in the liver was extracted using ISOGEN (Nippon Gene Co., Inc., Japan). Superscript ${ }^{\mathrm{TM}}$ III reverse transcriptase (Invitrogen, USA) was used for the synthesis of cDNA. Primers of the chemokines are shown in Table 1. Conditions for DNA amplications were set as follows: 26 cycles of DNA denaturation at $94^{\circ} \mathrm{C}$ for $1 \mathrm{~min}$, an annealing step at $56^{\circ} \mathrm{C}$ for $1 \mathrm{~min}$, and strand extension at $72^{\circ} \mathrm{C}$ for $45 \mathrm{sec}$. PCR amplication of $\alpha$-actin and its band intensity were used to determine the quality and quantity of the cDNA of the samples for semi-quantitative analyses.

Table 1. PCR Primers for chemokines and their receptors

\begin{tabular}{cll}
\hline & Primer sequence (forward) & Primer sequence (reverse) \\
\hline Chemokine & & \\
CCL-2 & AGCACCAGCCAACTCTCACT & TCTGGACCCATTCCTTCTTG \\
CCL-5 & CCCTCACCATCATCCTCACT & GGGATTACTGGAGTGGCATC \\
CX3CL-1 & GTTGGCTCCTGAGAGTGAGG & CAAAATGGCACAGACATTGG \\
\hline Chemokine receptor & \\
CCR-1 & GTTGGGACCTTGAACCTTGA & CTAGGACATTGCCCACCACT \\
CCR-2 & CCTGCAAAGACCAGAAGAGG & GATGGCCAAGTTGAGCAGAT \\
CCR-3 & TTCTACCGGCCCTCACATAC & CTGGGAACGTGTTGTTGATG \\
CCR-5 & ATTCTCCACACCCTGTTTCG & CGTTTGACCATGTGTTTCG \\
CX3CR-1 & CCTGCCTCTGAGAAATGGAG & ATCTCTCCAGCCCCTGAAAT \\
\hline
\end{tabular}




\section{In situ hybridization of CCL-2 and bright field immunohistochemistry of adjacent sections}

In situ hybridization (Lindeberg and Johansen, 1990) was conducted in order to determine sites of chemokine production. Complementary DNA probes were prepared through RT-PCR amplification using the primers as described previously in the protocols. PCR products were labeled with digoxigenin using a DIG high prime (Roche Inc., USA).

In situ hybridization was conducted using a DIG high prime detection starter kit (Roche Diagnostics Corp., USA) with modifications. Paraffin sections $(3 \mu \mathrm{m})$ of day 4 , day 7 and control livers were dewaxed and then immersed in $3 \%$ hydrogen peroxide in a methanol solution for $30 \mathrm{~min}$ to inactivate endogeneous peroxidase. They were then placed in $0.2 \mathrm{~N} \mathrm{HCl}$ for $10 \mathrm{~min}, 2 \mathrm{X}$ SSc $(300 \mathrm{mM}$ sodium chloride, $30 \mathrm{mM}$ sodium citrate, $\mathrm{pH} 7.0$ ) at $70^{\circ} \mathrm{C}$ for $30 \mathrm{~min}$, and then were treated with proteinase $\mathrm{K}$ (Nippon Gene) at $37^{\circ} \mathrm{C}$ for $1 \mathrm{~h}$. The sections were placed in an acetylation solution at RT for $10 \mathrm{~min}$. Hybridization probes were denatured and hybridization was conducted at $42{ }^{\circ} \mathrm{C}$ overnight. A sheep anti-DIG antibody (Roche Diagnostics, USA) was used at dilution of 1:500 at RT for $30 \mathrm{~min}$. Sections were rinsed and treated with peroxidase-conjugated donkey anti-sheep IgG (Jackson ImmunoResearch, USA) at RT for $1 \mathrm{~h}$. Peroxidase reaction was developed in a DAB reaction mixture. Serial paraffin sections were made for bright field immunohistochemistry.

\section{Statistical analyses}

Statistical analyses were conducted using the student $t$-test. P-values below 0.01 were regarded as statistically significant.

\section{Results}

\section{Induction of extramedullary hematopoiesis in the liver}

Extramedullary hematopoiesis was induced in the liver of the PHZ-treated splenectomized mice. No obvious sign of hematopoiesis was seen on day 4 (Fig. 3a). Numerous hematopoietic foci were identified chiefly distributed in the periportal regions by day 7 (Fig. 3b arrows). Electron microscopic analyses of the liver showed numerous hematopoietic foci, mostly consisting of erythroid cells at day 7 (Fig. 3c). A thin layer of endothelial cells surrounded most of the hematopoietic foci, indicating that the hematopoietic foci formed primarily intra-luminally within the expanded sinusoids (Fig. 3c arrowheads).

\section{Involvement of the RES cells during extramedullary hematopoiesis}

We used an intravital injection of fluorescent conjugated lectin (RCA-I) to delineate the sinusoidal endothelia and phagocytic cells of the liver according to our previous report (Nakamura-Ishizu et al., 2008). On day 4, multiple round cells, which exhibited a granular lectin staining pattern, were observed attached to the endothelia of central veins and sinusoids (Fig. $4 \mathrm{a}-\mathrm{d}$ ), whereas very few cells were attached to the endothelia of the vessels within the Glisson's sheath (i.e. hepatic arteries, and portal veins) (Fig. 4a). The cells attached to the central vein endothelia were positive for macrophage markers F4/80, CD11b, and LYVE-I (Fig. 4e-g). However, by day 7, multiple granular staining cells translocated into the hematopoietic foci in the periportal regions (Fig. $4 \mathrm{~h}-\mathrm{k}$ ). In contrast, very few cells were attached to the central vein on day 7 (Fig. 4h).

\section{Recruitment of PKH26 labeled blood monocytes into the liver for hematopoiesis}

In order to elucidate the source of recruited cells, PKH26 labeled peripheral blood monocytes from either PHZtreated or non-treated mice were transfused in various combinations as shown in Figure 2. Four hours after transplantation, PKH26 labeled monoctyes were observed in all samples regardless of the donor and recipient combination (Fig. $5 \mathrm{a}-\mathrm{c}$ ) though the degree of recruitment was significantly greater when the recipients had been treated with PHZ (S to P and P to P) (Fig. 6a). Three days after transplantation, only the PHZ treated recipients (S to $\mathrm{P}$ and $\mathrm{P}$ to $\mathrm{P}$ ) showed hematopoiesis (Fig. $5 \mathrm{~d}-\mathrm{f}$ ). No hematopoietic foci formation was noted in $\mathrm{P}$ to $\mathrm{S}$ or $\mathrm{S}$ to $\mathrm{S}$ samples. The hematopoietic foci in both samples contained PKH26 labeled cells. When double stained with CD45, a hematopoietic cell marker, PKH26 positive cells were in close contact with CD45 positive cells (Fig. $5 \mathrm{~g}-$ i). The percentage of foci containing the labeled cells was significantly higher in $\mathrm{P}$ to $\mathrm{P}$ samples than in $\mathrm{S}$ to $\mathrm{P}$ samples (Fig. 6b).

\section{Increased expression of chemokines and their receptors}

Chemokines are known to be involved in monocyte recruitment. (Rollins, 1997). In order to determine whether the infiltration of blood monocytes into 
Fig. 3. Induction of extramedullary hematopoirtic foci in the liver by PHZ. $\mathbf{a}$ and $\mathbf{b}$ : Hematoxylin-eosin staining of paraffin sections of the livers from PHZ-treated mice on day 4 (a) and day 7 (b). Arrows show hematopoietic foci in the periportal regions. c: An electron micrograph of a day 7 liver. Hematopoietic foci consist mostly of erythroid cells. Arrowheads show endothelial cells that have surrounded the hematopoietic foci. Bars: $30 \mu \mathrm{m}$ (a, b), $5 \mu \mathrm{m}$ (c)

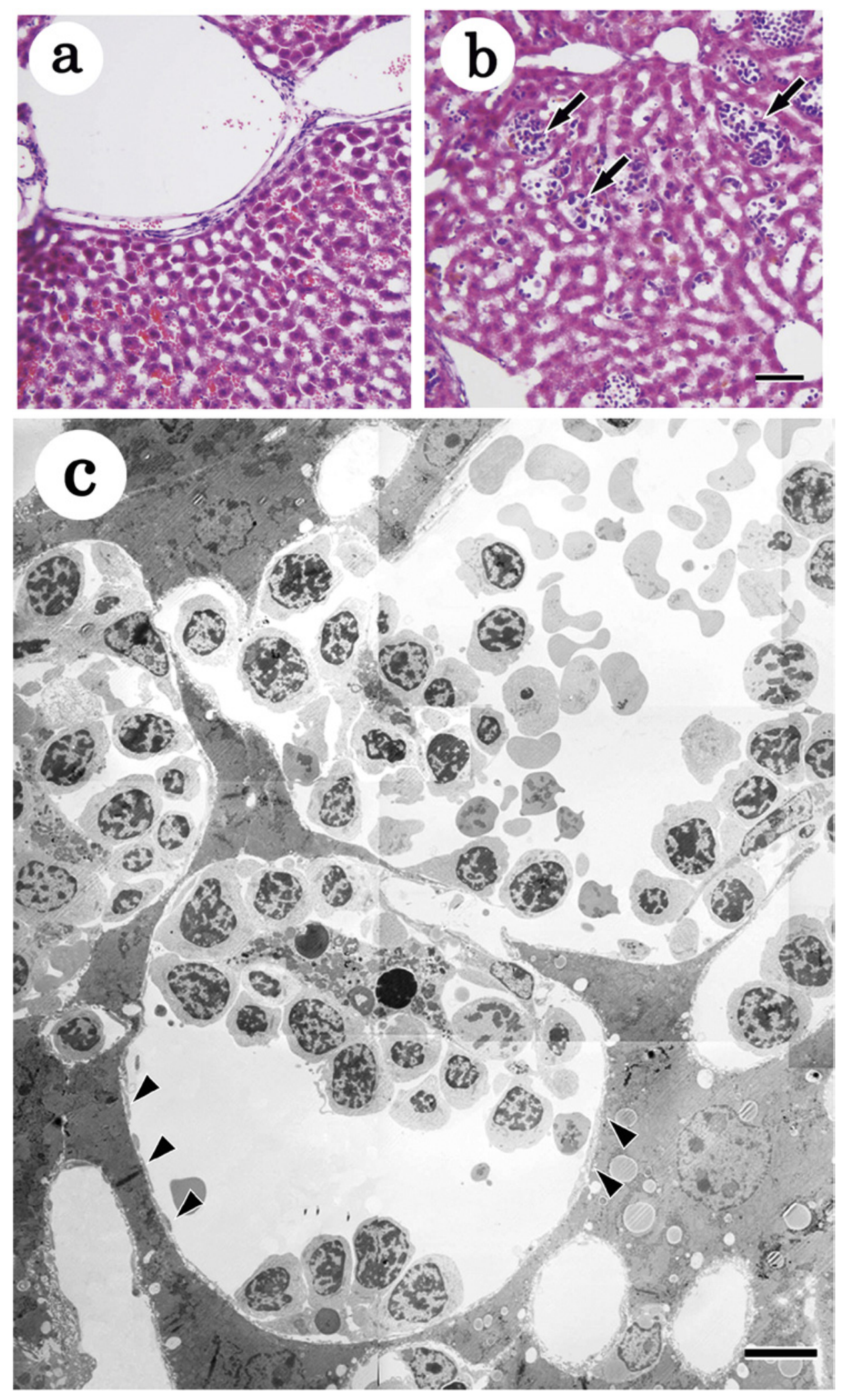


hematopoietic foci was triggered by some chemokines, we analyzed the expression of chemokines by semiquantitative RT-PCR. Semi-quantitative RT-PCR of days 2, 4 and 7 livers revealed an increase in CCL-2 and CCL-5 along with their receptors CCR-1, CCR-2, CCR-3, CCR-5 (Fig.7). An increase in the expression of chemokine CX3CL-1 and its receptor CX3CR-1 - which are known to be involved in the maintenance of tissue resident macrophages (Geissmann et al., 2003) — was also noted.

\section{In situ hybridization of chemokine CCL-2}

In situ hybridization of CCL-2 was performed to identify which cells produced these chemokines and receptors. In normal controls, no expression of CCL-2 was detected
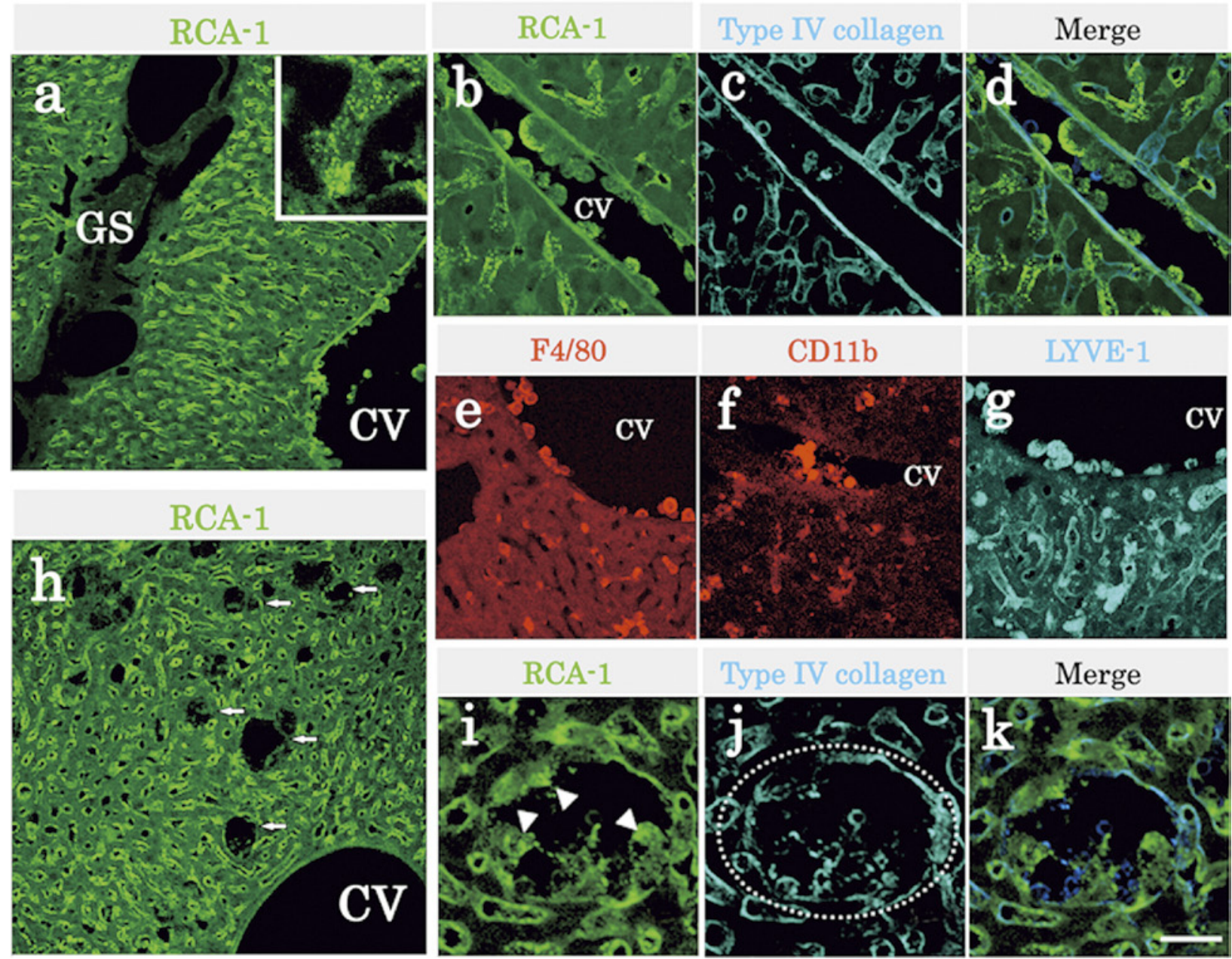

Type IV collagen

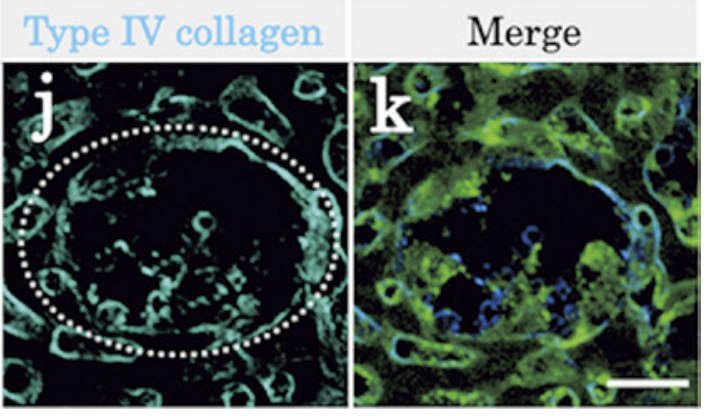

Fig. 4. Intravital lectin labeling and immunostaining of hematopoietic livers with various macrophage markers. a: Numerous round cells which stained granular to FITC-RCA-I (arrows) adhering to the central veins (CV) and portal areas of the liver on day 4, whereas very few lectin staining cells can be identified in the vessels within the Glisson's sheath (GS). b-d: Larger magnification of FITC-RCA-I staining cells (arrows) attached to the central vein on day 4. Type IV collagen (c) was stained to delineate the vascular structure. Staining of F4/80 (e), CD11b (f) and LYVE-1 (g) for day 4 samples. h: Low magnification of RCA-I stained BM on day 7. Arrows show hematopoietic foci. Note that no lectin staining cells are bound to the CV endothelia. i-k: High magnification of RCA-I (i), type IV collagen (j), and merged (k) staining images on day 7. RCA-I staining cells (arrowheads) reside in the hematopoietic foci (dotted circle). Bar: $25 \mu \mathrm{m}(\mathrm{a}, \mathrm{h}), 50 \mu \mathrm{m}(\mathrm{b}-\mathrm{g}, \mathrm{i}-\mathrm{k})$. 

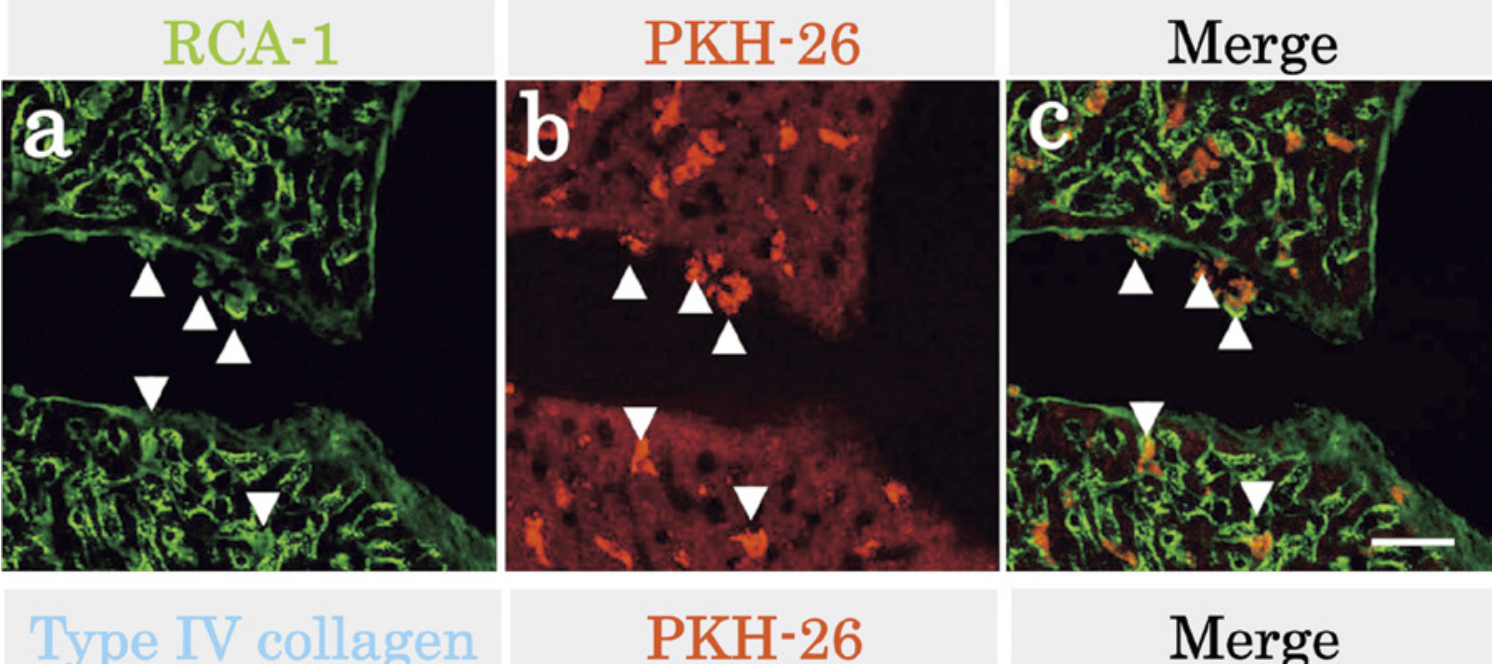

Merge
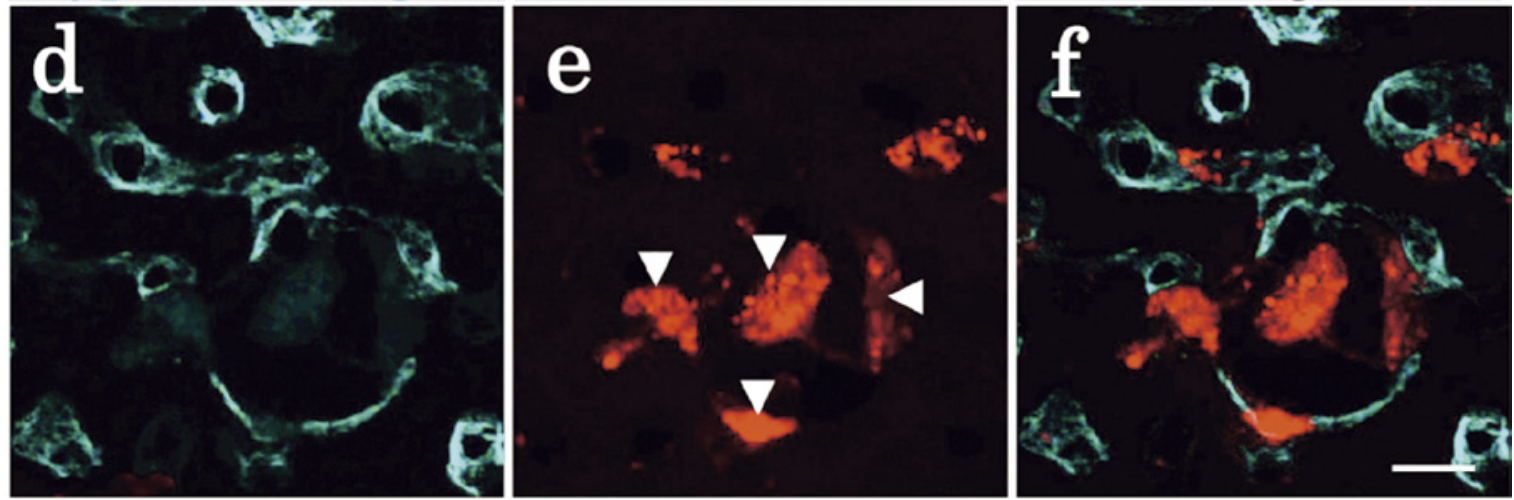

CD45

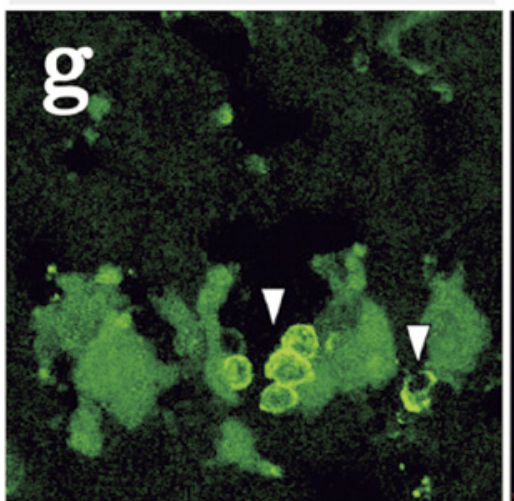

PKH-26

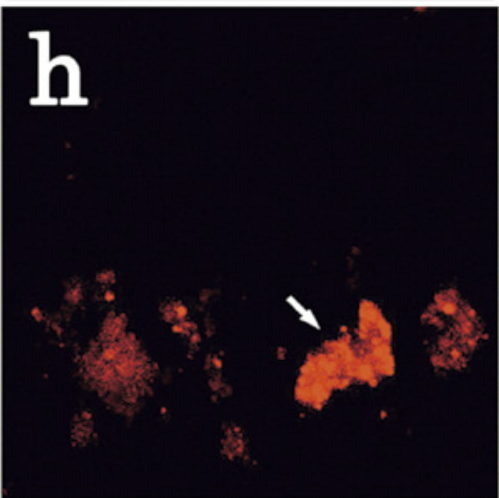

Merge

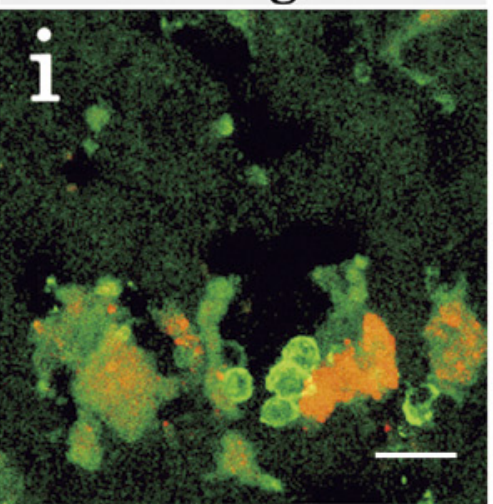

Fig. 5. Migration of PKH26 positive cells to the liver during extramedullary hematopoiesis. a-c: FITC-RCA-I (green) and PKH26 (red) positive cells (arrowheads) have infiltrated the liver $4 \mathrm{~h}$ after transplantation in a $\mathrm{P}$ to $\mathrm{P}$ combination. Bar: $75 \mu \mathrm{m}$. d-f: Hematopoietic foci of P to P samples, 3 days after transplantation. PKH26 (red) positive cells (arrowheads) accumulate in type IV collagen (blue) lined hematopoietic foci. g-i: Staining of CD45 (arrowheads) and PKH26 (arrow) on P to P samples. Bars: $50 \mu \mathrm{m}(\mathrm{d}-\mathrm{i})$ 

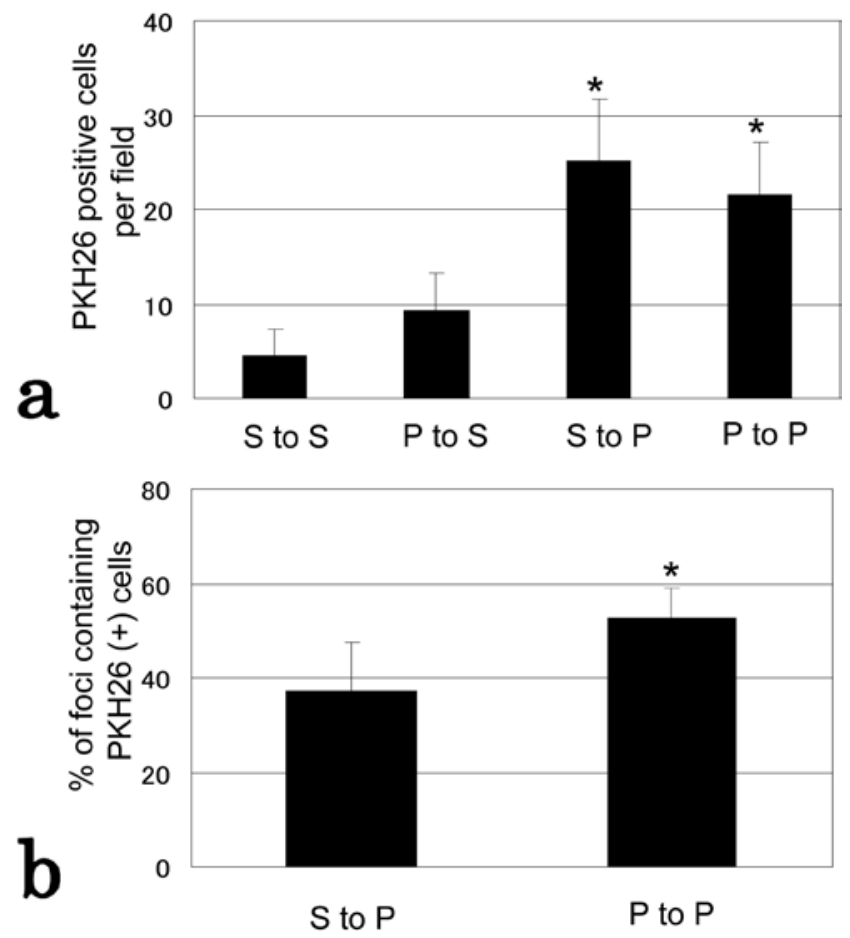

Fig. 6. Quantification and distribution of PKH26 positive cells in the liver. a: The number of PKH26 cells per field that were recruited into the liver (cells per X200 magnified field). Bars indicate standard errors of the means. Asterisks: $\mathrm{p}<0.01$ compared with $\mathrm{S}$ to $\mathrm{S}$. b: The percentage of foci that contained PKH26 positive cells in $\mathrm{S}$ to $\mathrm{P}$ and $\mathrm{P}$ to $\mathrm{P}$ samples. Bars indicate standard errors. Asterisk: $\mathrm{p}<0.01$

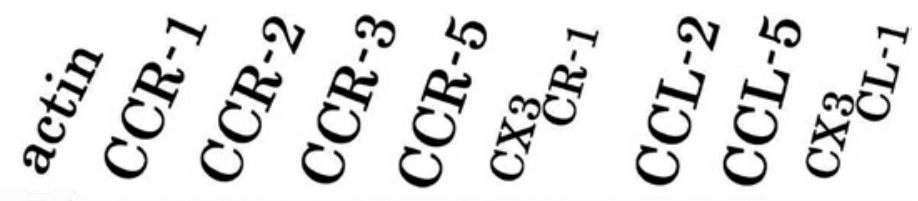

control

day 2

day 4
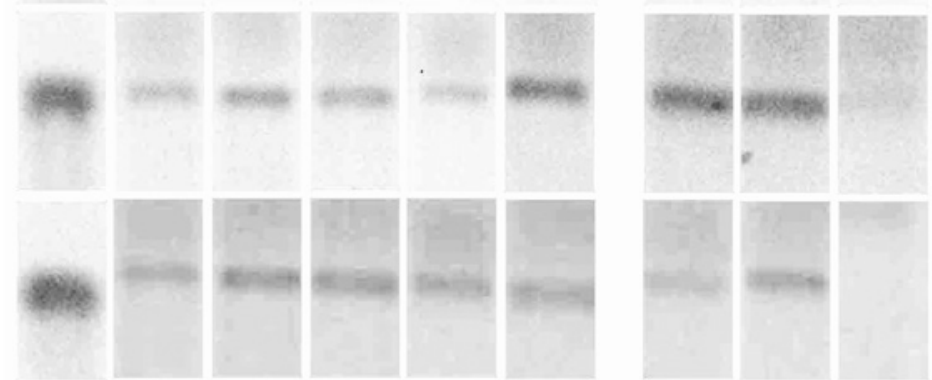

Fig. 7. Semi-quantitative PCR reaction of chemokines and chemokine receptors. PCR reaction of chemokine receptors CCR-1, CCR-2, CCR-3, and CCR-5 and their ligands. CCL-2 and CCL-5 have increased dramatically with hematopoiesis compared to controls. CX3CL-1 and day 7 its receptor CX3CR-1 also showed an increase in expression. 


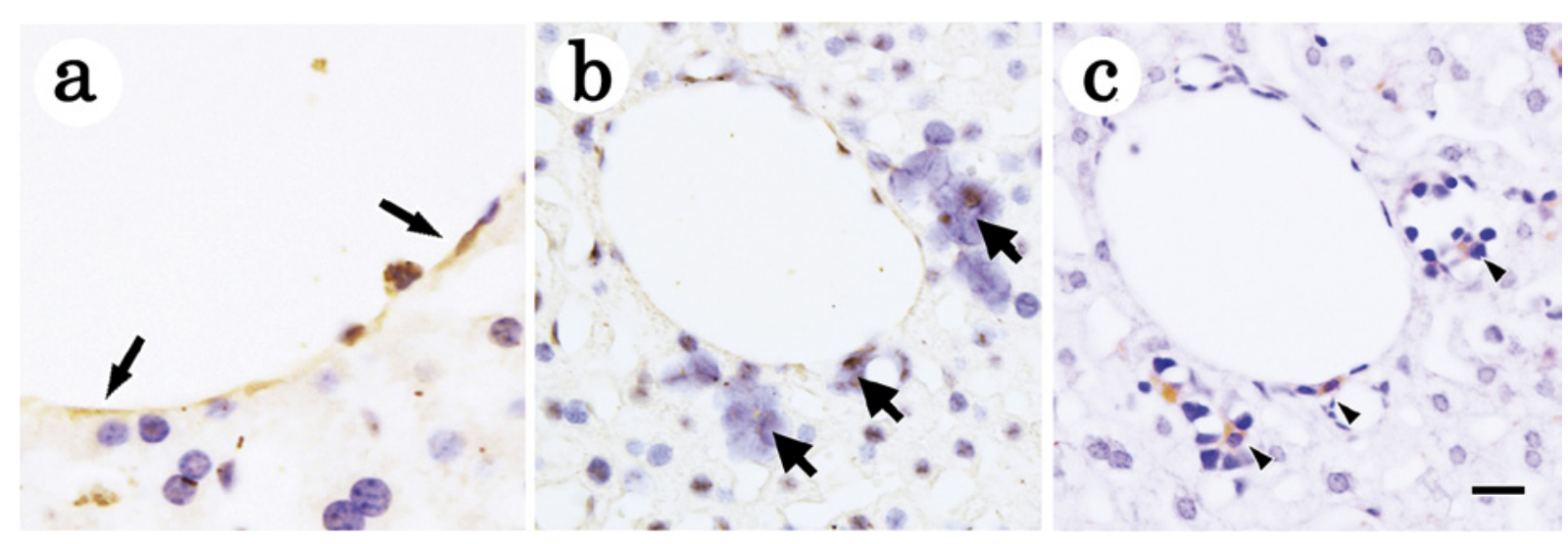

Fig. 8. In situ hybridization of chemokines CCL-2 and CCL-5, a: In situ hybridization of day 4 liver for CCL-2. b: ISH of day 7 livers for CCL-2. c: A serial adjacent section of $\mathbf{b}$ immuno-stained for F4/80, Brown stainings by DAB indicate the positivity for the chemokine mRNA on in situ hybridization sections (arrows). Brown stainings for DAB indicate positivity for F4/80 by immunohistochemistry (arrowheads). Counterstaining with hematoxylin. Note that the CCL-2 positive cells on b are positive for F4/80 on c. Bar: $10 \mu \mathrm{m}$

(data not shown). In the day 4 liver, CCL-2 (Fig.8a) were positive in the central vein endothelia. On day 7, CCL-2 production was noted on cells comprising the hematopoietic foci and endothelial cells (Fig 8b). Adjacent immunostained paraffin sections showed that F4/80 positive macrophages also produced CCL-2 on day 7 (Fig.8 b, c). CCL-2 expressions were not noted on the sinusoidal endothelial cells surrounding the hematopoietic foci.

\section{Discussion}

This study aimed to identify morphological and functional changes of the adult liver during extramedullary hematopoiesis. Using PHZ-treated splenectomized mice, we elucidated the role of recruited monocytes at the onset of and during extramedullary hematopoiesis in the liver. Our results identify and reveal characteristics of the extramedullary hematopoietic milieu of the liver which are comparable to that of the adult bone marrow yet are distinctive on their own.

We showed that monocytes of extra-hepatic origin are recruited into the liver to function as central macrophages. Prior to hematopoietic foci formation, numerous monocytes attach to the central vein endothelia and translocate to the proximity of the portal veins in the liver. Hematopoiesis induced by PHZ has been known to result in foci formation in the periportal regions (VidalVanaclocha et al., 1990), with which our results concur.
A thin layer of endothelial cells surrounded most of the hematopoietic foci, which suggested that hematopoietic foci formed primarily intra-luminally within the expanded sinusoids. Also, in situ hybridization analyses confirmed that the central vein endothelia produced the monocyte attracting chemokines CCL- 2 and CCL- 5 on day 4 prior to hematopoietic foci formation. These monocytes were thought to migrate in a retrograde direction from the central veins towards the portal areas for hematopoiesis. Transplanted PKH26-labeled peripheral blood monocytes were incorporated in the hematopoietic foci and were in close contact with hematopoietic cells, seemingly nursing these cells until maturation. Some reports have identified lymphoid cells as migrating into the liver during extramedullary hematopoiesis (Mori et al., 1987); however, reports like ours that specify the direct migration and involvement of extra-hepatic monocytes are without precedent. The exact origin of these peripheral blood monocytes and their precise mechanism of activation to induce and support hematopoiesis remain unclear and should be further investigated.

Not only the recruited monocytes but also the resident cellular components were necessary for extramedullary hematopoiesis. The PKH26 transplantation showed that the PHZ-treated liver increased its potential to recruit peripheral monocytes. Still, not all hematopoietic foci contained recruited PKH26 positive cells. Moreover, no foci formed in $\mathrm{P}$ to $\mathrm{S}$ or $\mathrm{S}$ to $\mathrm{S}$ transplants, showing that recruited monocytes alone cannot initiate hematopoiesis. Semi-quantitative PCR showed an increase in CCL-2 and 
CCL-5, which mainly involve the recruitment of activated monocytes during various processes such as inflammation (Mantovani et al., 2004). On the other hand, we also noted an increase in CX3CL-1 and its receptor that homeostatically recruit resident macrophages (Geissmann et al., 2003). This suggested that both recruited monocytes and resident macrophages participated in liver hematopoiesis.

Our data also indicated that chemokines may be involved in later stages of extramedullary hematopoiesis. Even after initial monocyte recruitment, PCR data showed a persistent chemokine production. Day 7 in situ hybridization confirmed that $\mathrm{F} 4 / 80$ positive macrophages in the hematopoietic foci produced CCL-2 which accentuated the relevance of these chemokines during hematopoiesis. However, the degree and the precise mechanisms through which these chemokines act need further investigation. Chemokine CCL-3 (MIP$1 \alpha)$ and its receptors act as regulators of bone marrow hematopoiesis (Broxmeyer and Kim, 1999). The intense expression of CCL-2 and CCL-5 within the hematopoietic foci suggests that these chemokines and their receptors may play a role in the regulation of extramedullary hematopoiesis.

In conclusion, the recruitment of extra-hepatic monocytes is a vital process during extramedullary hematopoisis of the liver. The local and extra-hepatic monocytes compromise a milieu for extramedullary hematopoiesis in the liver. Ontogenetically, macrophages carry primitive characteristics and are the first cells that respond to pathological conditions such as inflammation (Anastassova-Kristeva, 2003). Indeed, macrophages play a pivotal role in recruiting immature cells during angiogenesis and tumor metastasis (Lewis and Pollard, 2006). They have thus been recently recognized as an important participant of the pre-metastatic niche for tumor progression and invasion (Mantovani et al., 2006). Assuming that monocyte activation preceded hematopoiesis, our observations provide additional insight into characterizing these cells as relevant supporting cells in extramedullary hematopoiesis.

\section{Acknowledgements}

The authors are grateful to Ms. Y. Yamazaki, Ms. K. Nakada, Ms. H. Sagawa, and Ms. K. Motomaru for their devoted technical help.

\section{References}

Adler SS, Trobaugh FE Jr: Hemopoietic support capacity of the adult mouse liver: II. Studies in acetylphenylhydrazine-treated mice. Am J Hematol 4: 325-335 (1978).

Ahmed A, Chang CC: Chronic idiopathic myelofibrosis: clinicopathologic features, pathogenesis, and prognosis. Arch Pathol Lab Med 130: 1133-1143 (2006).

Anastassova-Kristeva M: The origin and development of the immune system with a view to stem cell therapy. $J$ Hematother Stem Cell Res 12: 137-154 (2003).

Broxmeyer HE, Kim CH: Regulation of hematopoiesis in a sea of chemokine family members with a plethora of redundant activities. Exp Hematol 27: 1113-1123 (1999).

Chasis JA: Erythroblastic islands: specialized microenvironmental niches for erythropoiesis. Curr Opin Hematol 13: 137-141 (2006).

de Jong JP, Leenen PJ, Voeman JS, van der Sluijs-Gelling AJ, Ploemacher RE: A monoclonal antibody (ERHR3) against murine macrophages. II. Biochemical and functional aspects of the ER-HR3 antigen. Cell Tissue Res 275: 577-585 (1994).

Geissmann F, Jung S, Littman DR: Blood monocytes consist of two principal subsets with distinct migratory properties. Immunity 19: 71-82 (2003).

Hanspal M, Hanspal JS: The association of erythroblasts with macrophages promotes erythroid proliferation and maturation: a $30-\mathrm{kD}$ heparin-binding protein is involved in this contact. Blood 84: 3494-3504 (1994).

Klein I, Cornejo JC, Polakos NK, John B, Wuensch SA, Topham DJ, Pierce RH, Crispe IN: Kupffer cell heterogeneity: functional properties of bone marrow derived and sessile hepatic macrophages. Blood 110: 4077-4085 (2007).

Kopp H-G., Avecilla ST, Hooper AT, Rafii S: The bone marrow vascular niche: home of HSC differentiation and mobilization. Physiology 20: 349-356 (2005).

Lee SH, Crocker PR, Westaby S, Key N, Mason DY, Gordon S, Weatherall DJ: Isolation and immunocytochemical characterization of human bone marrow stromal macrophages in hemopoietic clusters. $J$ Exp Med 168: 1193-1198 (1988).

Lewis CE, Pollard JW: Distinct role of macrophages in different tumor microenvironments. Cancer Res 66: 605-612 (2006). 
Lindeberg H, Johansen L: The presence of human papillomavirus (HPV) in solitary adult laryngeal papillomas demonstrated by in-situ DNA hybridization with sulphonated probes. Clin Otolaryngol Allied Sci 15: 367-371 (1990).

Mantovani A, Schioppa T, Porta C, Allavena P, Sica A: Role of tumor-associated macrophages in tumor progression and invasion. Cancer Metastasis Rev 25: 315-322 (2006).

Mantovani A, Sica A, Sozzani S, Allavena P, Vecchi A, Locati M: The chemokine system in diverse forms of macrophage activation and polarization. Trends Immunol 25: 677-686 (2004).

Mori M, Kirizuka K, Sadahira Y, Nakamoto S, Seno S: Early events in extramedullary hemopoiesis in the liver: lymphoid cell migration into the liver. Nippon Ketsueki Gakkai Zasshi 50: 1-6 (1987).

Nakamura-Ishizu A, Morikawa S, Shimizu K, Ezaki T: Characterization of sinusoidal endothelial cells of the liver and bone marrow using an intravital lectin injection method. J Mol Histol 39: 471-479 (2008).

O'Keane JC, Wolf BC, Neiman RS: The pathogenesis of splenic extramedullary hematopoiesis in metastatic carcinoma. Cancer 63: 1539-1543 (1989).

Penn PE, Jiang DZ, Fei RG, Sitnicka E, Wolf NS: Dissecting the hematopoietic microenvironment. IX. Further characterization of murine bone marrow stromal cells. Blood 81: 1205-1213 (1993).

Rollins BJ: Chemokines. Blood 90: 909-928 (1997).
Schofield R: The relationship between the spleen colonyforming cell and the haemopoietic stem cell. Blood Cells 4: 7-25 (1978).

Taher A, Isma'eel H, Cappellini MD: Thalassemia intermedia: revisited. Blood Cells Mol Dis 37: 12-20 (2006).

Taichman RS: Blood and bone: two tissues whose fates are intertwined to create the hematopoietic stem-cell niche. Blood 105: 2631-2639 (2005).

Vidal-Vanaclocha F, Alonso-Varona A, Avala R, Bovano MD, Barberá-Guillem E: Coincident implantation, growth and interaction sites within the liver of cancer and reactive hematopoietic cells. Int J Cancer 46: 267271 (1990).

Weiss L: The hematopoietic microenvironment of the bone marrow: an ultrastructural study of the stroma in rats. Anat Rec 186: 161-184 (1976).

Wright DE, Wagers AJ, Gulati AP, Johnson FL, Weissman IL: Physiological migration of hematopoietic stem and progenitor cells. Science 294: 1933-1936 (2001).

Yin T, Li L: The stem cell niches in bone. J Clin Invest 116: 1195-1201 (2006).

Zuckerman KS, Rhodes RK, Goodrum DD, Patel VR, Sparks B, Wells J, Wicha MS, Moyo LA: Inhibition of collagen deposition in the extracellular matrix prevents the establishment of a stroma supportive of hematopoiesis in long-term murine bone marrow cultures. J Clin Invest 75: 970-975 (1985). 